\title{
Erratum to: Saturation Approach in Top Production ${ }^{1}$ \\ G. R. Boroun*, ** \\ Department of Physics, Razi University, Kermanshah, 67149 Iran \\ *e-mail: grboroun@gmail.com \\ **e-mail: boroun@razi.ac.ir \\ Submitted November 5, 2018; accepted for publication November 6, 2018
}

DOI: $10.1134 /$ S1547477119010126

The affiliation should read:

Department of Physics, Razi University, Kermanshah, 67149 Iran

The original article can be found online at https://doi.org/10.1134/S1547477118040064.

\footnotetext{
${ }^{1}$ The article is published in the original.
} 